\title{
Corn Gluten Hydrolysate Affects the Time-Course of Metabolic Changes Through Appetite Control in High-Fat Diet-Induced Obese Rats
}

\author{
Hyojung Lee', Hyo Jin Lee', Ji Yeon Kim ${ }^{2, *}$, and Oran Kwon ${ }^{1, *}$
}

This study first investigated the effects of corn gluten hydrolysate (CGH) (1.5 g/day) administration for 7 days on appetite-responsive genes in lean Sprague-Dawley (SD) rats. In a second set of experiments, the metabolic changes occurring at multiple time points over 8 weeks in response to CGH (35.33\% wt/wt) were observed in high-fat (HF, $60 \%$ of energy as fat) diet-fed SD rats. In lean rats, the hypothalamus neuropeptide- $Y$ and proopiomelanocortin mRNA levels of the CGH group were significantly changed in response to $\mathrm{CGH}$ administration. In the second part of the study, CGH treatment was found to reduce body weight and perirenal and epididymal fat weight. CGH also prevented an increase in food intake at 2 weeks and lowered plasma leptin and insulin levels in comparison with the HF group. This reduction in the plasma and hepatic lipid levels was followed by improved insulin resistance, and the beneficial metabolic effects of CGH were also partly related to increases in plasma adiponectin levels. The Homeostasis Model of Assessment - Insulin Resistance (HOMA-IR), an index of insulin resistance, was markedly improved in the HFCGH group compared with the HF group at 6 weeks. According to the microarray results, adipose tissue mRNA expression related to G-protein coupled receptor protein signaling pathway and sensory perception was significantly improved after 8 weeks of CGH administration. In conclusion, the present findings suggest that dietary CGH may be effective for improving hyperglycemia, dyslipidemia and insulin resistance in diet-induced obese rats as well as appetite control in lean rats.

\footnotetext{
${ }^{1}$ Department of Nutritional Science and Food Management, Ewha Womans University, Seoul 120-750, Korea, ${ }^{2}$ Department of Food Science and Technology, Seoul National University of Science and Technology, Seoul 139-743, Korea

*Correspondence: jiyeonk@seoultech.ac.kr (JYK); orank@ewha.ac.kr (OK)

Received 23 April, 2015; revised 30 August, 2015; accepted 18 September, 2015; published online 6 November, 2015
}

Keywords: corn gluten hydrolysates, food intake, leptin, lipid metabolism, obesity

\section{INTRODUCTION}

Obesity is a complicated illness status attributed to genetic factors, dysfunction in the hypothalamus, and unbalanced food intake and energy expenditure (Das, 2010). This illness status is usually associated with dyslipidemia and insulin resistance, which are components of metabolic syndrome, as well as with chronic low-grade inflammation (Calder et al., 2011). Considering the rapid growth in the prevalence ratio for obesity and associated disease, identifying food components that are effective in preventing obesity development has attracted interest. Plant protein and dietary fiber have been identified as food constituents associated with decreased appetite and body weight, although data from human trials are still equivocal (Clark and Slavin, 2013; Slavin, 2013; Slavin and Green, 2007; Wanders et al., 2011). The identification of food constituents that prevent obesity may be achieved by using laboratory animals over which there is complete control of the diet and by collecting liver or adipose tissues over time. Moreover, this approach allows researchers to explore underlying molecular or biochemical mechanisms. Studies in rats have shown that diets containing dietary fiber or protein result in lower body weight and/or adiposity (Adam et al., 2014; Aoyama et al., 2000; Davis et al., 2005).

Corn is known to be a good source of branched amino acids such as leucine, which is an essential amino acid with a unique role in protein synthesis (Layman, 2003; Layman and Walker, 2006). Previously, we compared the anti-obesity effect of soy, wheat and corn protein. Among them, corn gluten hydrolysate $(\mathrm{CGH})$ was associated with body weight reduction after 8 weeks of administration in high-fat diet-induced obese (DIO) rats (Kim et al., 2009). Another study was conducted on branched amino acids such as leucine, as CGH contains high levels of free branched amino acids compared with other plant protein sources. Leucine, as well as CGH administration, decreased body weight, plasma leptin, fat mass, and food intake after 8 weeks of administration (Bong et al., 2010). In another study, 4 weeks of CGH administration significantly improved body weight, body fat, plasma leptin and hepatic lipid levels (Mun et al., 2014). Thus, upon observing the effects of CGH on DIO rats, we repeatedly found that CGH significantly decreased the food intake level.

The maintenance of proper body weight through appetite control is a complex process regulated by redundant and distri- 
buted neural systems that integrate a multitude of cognitive and homeostatic responses to accurately regulate systemic energy balance. The signals obtained from hypothalamus are critically related to detect circulating and local components that messenger information regarding the energy status of the organism (Schneeberger et al., 2014).

Therefore, based on the previous findings, we hypothesized that $\mathrm{CGH}$ administration decreases food intake and plasma leptin levels during the early stage of DIO via interactions with mediators of leptin's actions in the hypothalamus. In the present study, to obtain mechanistic insight on appetite, we examined the effect of short-term intake of CGH on hypothalamus mRNA levels in lean rats. Furthermore, the time-course effects of $\mathrm{CGH}$ administration on biochemical metabolic changes were investigated in the DIO rat at 2-week intervals over the course of 8 weeks, and the resulting transcriptional changes in adipose tissue in $\mathrm{CGH}$ rats treated for 8 weeks were compared with those of high-fat-fed control rats.

\section{MATERIALS AND METHODS}

\section{Preparation of $\mathrm{CGH}$}

CGH was manufactured using Alcalase, Protamex, and Flavourzyme (Novo Nordisk, Denmark) by Sempio Food Company (Korea). The composition of CGH is as follows: $67.4 \%$ protein, $25.64 \%$ carbohydrate, $4 \%$ ash, $97.04 \%$ solid content, $2.96 \%$ moisture, $10.2 \%$ total nitrogen, $39.28 \%$ free amino acid, and $3.51 \%$ water.

\section{Animals and diets}

Eight-week-old male SD rats were obtained from G-Bio Animals Inc. (Korea). All of the SD rats were individually housed in stainless steel wire-mesh cages at a temperature of $22-24^{\circ} \mathrm{C}$ with a $12 \mathrm{~h}$ light/dark cycle and $45 \pm 5 \%$ humidity. Experiment 1 examined the short-term effects of $\mathrm{CGH}$ on food intake and hypothalamus mRNA expression using normal lean rats. After one week of acclimatization, the rats (average weight $287.26 \pm 2.16$ g) were randomized into 2 groups according to body weight. CGH solution in water $(1.5 \mathrm{~g} /$ day) or vehicle was administered by gavage for 7 days ( $n=7$ per each group). Rats were fed a commercial pellet diet (Orient Bio Inc., Korea), and water was provided ad libitum. Food intake was measured daily from each cage. Experiment 2 examined the time course of metabolic changes in rats fed $\mathrm{CGH}$ as a protein source in a high-fat diet. All rats were randomly divided into three groups according to body weight and fed the designated induction diet for 13 weeks. The normal-fat diet consisted of AIN-93G (NF, 17\% of energy as fat; $n=25$ ), and the high-fat diet was modified from the AIN$93 \mathrm{G}$ diet $(\mathrm{HF}, 60 \%$ of energy as fat; $n=50)$. After 13 weeks, the HF group was re-allocated into 2 groups according to body weight and fed the designated experimental diet for 8 weeks (Table 1): high-fat diet (HF, $n=25)$ or high-fat diet with $35.33 \%$

Table 1. Composition of experimental diet (Unit: $\mathrm{g} / \mathrm{kg}$ diet)

\begin{tabular}{lccc}
\hline & & Group & \\
& Ingredients & HF & HF-CGH \\
\cline { 2 - 3 } & NF & 0.00 & 0.00 \\
Corn starch & 397.49 & 149.76 & 63.00 \\
Dextrinized corn starch & 132.00 & 102.11 & 102.11 \\
Sucrose & 100.00 & 0.00 \\
Casein (>85\% protein) & 200.00 & 272.29 & 353.33 \\
Corn gluten hydrolysate (CGH) & 0.00 & 0.00 & 101.0 \\
Soybean Oil & 70.00 & 230.0 \\
Lard & 0.00 & 243.70 & 60.0 \\
Fiber & 50.00 & 68.07 & 41.0 \\
Mineral mix & 35.00 & 47.65 & 12.0 \\
Vitamin mix & 10.00 & 13.61 & 3.5 \\
L-Cystine & 3.00 & 4.08 & 2.9 \\
Choline bitartrate & 2.50 & 3.40 & 0.0 \\
tert-Butylhydroquinone & 0.01 & 0.02 & $1,000.0$ \\
Total amount & $1,000.0$ & $1,000.0$ & 5168.29 \\
Total Calorie (kcal) & 3737.61 & 5088.80 & 21 \\
Carbohydrates (\% as kcal) & 64 & 21 & 19 \\
Protein (\% as kcal) & 19 & 19 & 60 \\
Fat (\% as kcal) & 17 & 60 &
\end{tabular}

${ }^{1)}$ Control, AIN-93G diet; NF, normal fat control; HF, high fat control; HF-CGH, high fat with CGH as a protein source

${ }^{2)}$ Mineral mix (AIN-93G) (g/kg mixture): anhydrous calcium carbonate 357, monobasic potassium phosphate 196, sodium chloride 74 , potassium sulfate 46.6 , tripotassium citrate monohydrate 70.78 , magnesium oxide 24 , ferric citrate 6.06 , zinc carbonate 1.65 , manganese carbonate 0.63 , cupric carbonate 0.3 , potassium iodate 0.01 , anhydrous sodium selenate 0.01025 , ammoniumparamolybdate 4 -hydrate 0.00795 , sodium metasilicate 9 -hydrate 1.45 , chromium potassium sulfate 12 -hydrate 0.0275 , boric acid 0.0815 , sodium fluoride 0.0635 , nickel carbonate 0.0318 , lithium chloride 0.0714 , ammonium vanadate 0.0066 , powdered sucrose 221.026

${ }^{3)}$ Vitamin mix (AIN-93-VX) (g/kg mixture): nicotinic acid 3, Ca-pantothenate 1.6, pyridoxine $\mathrm{HCl} 0.7$, thiamin- $\mathrm{HCl} 0.6$, riboflavin 0.6, folic acid 0.2 , D biotin 0.02 , vitamin B12 (0.1\% cyanocobalamin in mannitol) 2.5, vitamin E (all-ras- $\alpha$-tocopheryl acetate, 500 IU/g) 15 , vitamin A (alltrans-retinyl palmitate, $500,000 \mathrm{IU} / \mathrm{g}$ ) 0.8 , vitamin D3 (cholecalciferol, $400,000 \mathrm{IU} / \mathrm{g}$ ) 0.25 , vitamin $\mathrm{K}$ (phylloquinone) 0.075 , powdered sucrose 974.655 
(w/w) CGH (HF-CGH, n = 25). To investigate the time-course effects of CGH over 8 weeks, 5 rats from the NF, HF, and HF$\mathrm{CGH}$ groups were randomly selected based on their body weight and sacrificed biweekly at $0,2,4,6$ and 8 weeks $(n=5$, per group). The experimental protocol was approved by the Institutional Animal Care and Use Committee (IACUC) of the Ewha Womans University of Korea (Reference Number: 201021-2 and 2012-01-034).

\section{Specimen collection}

At the end of each experimental period, all rats were sacrificed after $12 \mathrm{~h}$ of fasting. Whole blood was drawn from the heart and transferred to polypropylene tubes containing both ethylene diamine tetra-acetic acid (EDTA) and $3.2 \%$ sodium citrate and then centrifuged at $2,800 \mathrm{rpm}$ for $30 \mathrm{~min}$ at $4^{\circ} \mathrm{C}$ (Union $32 \mathrm{R}$ PLUS, Han-il, Korea). In experiment 1, the hypothalamus was removed and frozen at $-80^{\circ} \mathrm{C}$ until analysis. In experiment 2 , the liver and epididymal and perirenal fat pads were removed, weighed and frozen at $-80^{\circ} \mathrm{C}$ until analysis.

\section{Biochemical assays}

The fasting plasma glucose concentration was measured with the glucose oxidase method (Cayman Chemical, USA). Plasma insulin was determined using a rat insulin enzyme-linked immunosorbent assay (ELISA) kit (Mercodia, Sweden). The HOMA-IR level was calculated as fasting plasma glucose $(\mathrm{mmol} / \mathrm{L}) \times$ fasting plasma insulin $(\mu \mathrm{lU} / \mathrm{ml})$ divided by 22.5 . The plasma leptin level was measured using a radioimmunoassay kit (Invitrogen, USA). The concentration of plasma adiponectin was analyzed with an ELISA kit (B-Bridge, Japan). All procedures were performed in accordance with the manufacturer's instructions. Plasma triglyceride (TG) and total-cholesterol (Total-C) levels were analyzed using commercial enzymatic kits (Asan Pharmaceutical, Korea) based on a modification of the lipase-glycerol phosphate oxidase and the cholesterol oxidase methods (Caudill et al., 1998; Panteghini et al., 1993). The HDL-C level was measured using a kit (Asan Pharmaceutical, Seoul, Korea) after precipitation of the lipoproteins. The hepatic lipids were extracted using Bligh's method (Bligh and Dyer, 1959). Liver tissue $(0.5 \mathrm{~g})$ was homogenized in $1.5 \mathrm{ml} 0.9 \%$ saline, and $7.5 \mathrm{ml}$ of a $1: 2(\mathrm{v} / \mathrm{v})$ chloroform-methanol $(1: 2, \mathrm{v} / \mathrm{v})$ solution was added to the homogenate. After centrifugation at $3,000 \mathrm{rpm}$ for $20 \mathrm{~min}$, the lower aqueous phase was decanted into a fresh tube and the remaining upper phase was mixed thoroughly. The extraction and washing procedure was repeated twice, the extracts were pooled, and $10 \mathrm{~g}$ sodium sul- fate was added to the lipid extract. The extract was then filtered using Whatman filter paper (GE Healthcare, Korea) soaked in chloroform and dried to a constant weight in an oven at $50^{\circ} \mathrm{C}$. The lipid extract was dissolved in $5 \mathrm{ml}$ ethanol containing $0.5 \%$ Triton X-100, and the analyses were conducted with the same enzymatic kit used in the plasma analyses. After extraction, Hepatic, Total-C and total lipids were measured with the same methods used to determine plasma lipids.

Quantitative real-time reverse transcriptase polymerase chain reaction (RT-PCT) assay

Total RNA from the homogenized hypothalamus was extracted according to the manufacturer's instruction using TRIzol (Invitrogen, USA). The total RNA concentration and 260/280 nm ratio were evaluated using a spectrophotometer (BioSpec-nano, Shimadzu Corp., Japan). Only samples with a 260/280 nm ratio between 1.7 and 2.1 were further processed. Single-strand cDNA was synthesized from $2 \mu \mathrm{g}$ of total RNA using the High Capacity RNA-to-cDNA Kit (Applied Biosystems, USA). Quantitative RT-PCR was performed using the TaqMan method in the Step-One-Plus RT-PCR System (Applied Biosystems, USA) The primer sets for target genes in the rat hypothalamus included the leptin receptor (LEPR; Rn01433205_m1), neuropeptide-Y (NPY; Rn01410145_m1), pro-opiomelanocortin (POMC; Rn00595020 m1), and $\bar{\beta}$-actin (ACTB; Rn00667869 m1). Amplification was initiated with a 10-min template denaturation step at $95^{\circ} \mathrm{C}$ followed by 40 cycles at $95^{\circ} \mathrm{C}$ for $15 \mathrm{~s}$ and $60^{\circ} \mathrm{C}$ for $1 \mathrm{~min}$. The relative amounts of these mRNAs were normalized to the amount of $\beta$-actin, and the relative amount of all RNA was calculated using the comparative $\mathrm{C}_{\mathrm{T}}$ method.

\section{Microarray assay and functional annotation analysis} After experiment 2 was completed, total RNA was extracted from white adipose tissue (WAT) using the TRI REAGENT (Molecular Research Center, USA) according to the manufacturer's instructions. Each total RNA sample $(10 \mu \mathrm{g})$ was labeled and hybridized for $12 \mathrm{~h}$ at $42^{\circ} \mathrm{C}$ in a MAUl system (BioMicro Systems, Inc., USA). The hybridized slides were washed and scanned. The Roche NimbleGen Rat genome 12-plex array was submitted to Roche NimbleGen Inc. for microarray design and manufacture using maskless, digital micromirror technology (Roche NimbleGen, Inc., USA). Twelve replicates of the genome were included per chip. An average of 5 different 60base oligonucleotides (60-mer probes) represented each gene in the genome. A quality control check (hybridization) was performed for each array, which contained on-chip control oligo-
$\boldsymbol{A}$

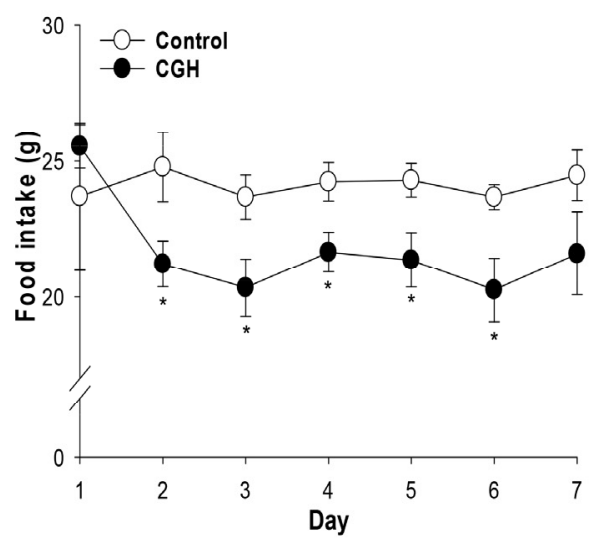

B

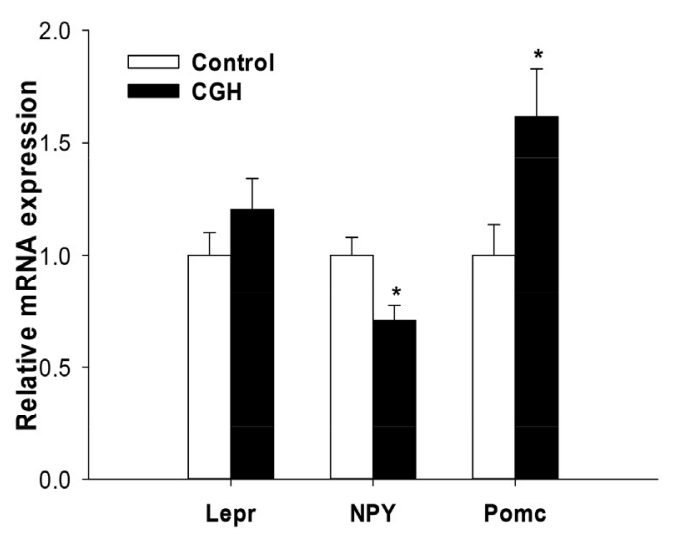

Fig. 1. Effects of $\mathrm{CGH}$ on food intake $(A)$ and hypothalamic mRNA levels $(B)$ in lean rats. $\mathrm{CGH}$ was fed as a protein source for 1 week. Normal diet was used as a control diet. Values are expressed as means \pm SE. Significant differences between two groups were analyzed with Student's $t$-test $\left({ }^{*} P<0.05\right)$. 
$\boldsymbol{A}$

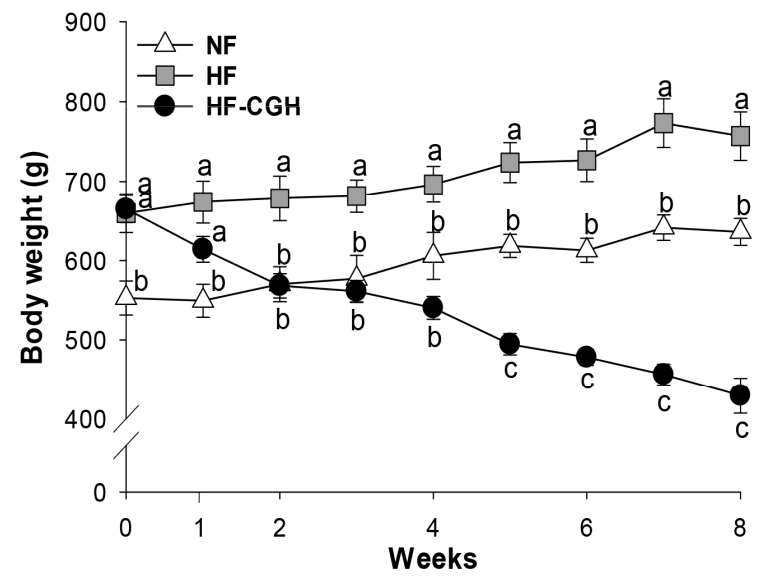

B

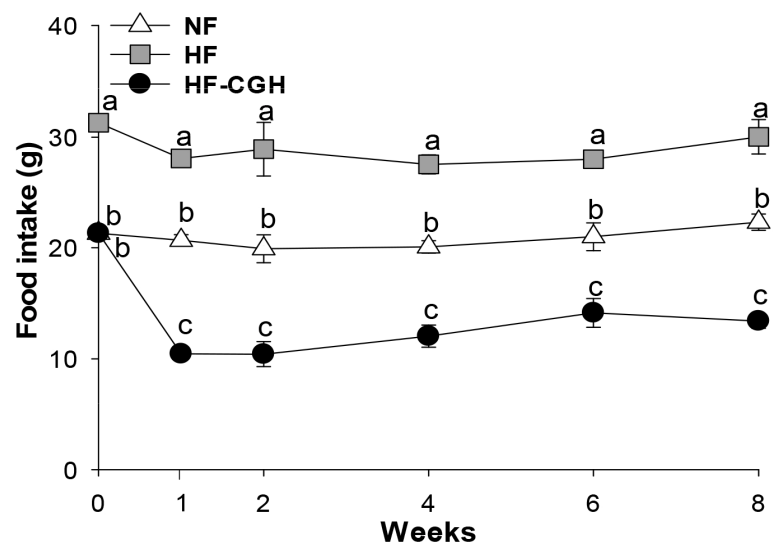

Fig. 2. Body weight $(A)$ and food intake $(B)$ profiles during an 8-week CGH diet in DIO rats. Eight-week-old male SD rats were randomly divided into three groups according to body weight and fed the designated induction diet for 13 weeks; the normal-fat diet consisted of the AIN$93 \mathrm{G}$ diet $(\mathrm{NF}, \mathrm{n}=25)$, and the high-fat diet was modified from the AIN-93G diet $(\mathrm{HF}, \mathrm{n}=50)$. After 13 weeks, the HF group was re-randomized into 2 groups according to body weight and fed the designated experiment diet for 8 weeks: high-fat diet $(H F, n=25)$ or high-fat diet with $C G H$ (HF-CGH, $n=25)$. Body weight and food intake were measured each week. Values are expressed as means \pm SE. Significant differences among the three groups were analyzed by ANOVA. Values showing a different superscript letter among the three diet groups are significantly different $(P<0.05)$.

nucleotides. The arrays were analyzed using an Axon GenePix 4000B scanner with associated software (Molecular Devices Corp., USA). Gene expression levels were calculated with NimbleScan Version 2.4 (Roche NimbleGen, Inc., USA). Relative signal intensities for each gene were generated using the Robust Multi-Array Average algorithm. The data were processed based on a median polish normalization method using the NimbleScan Version 2.4 (Roche NimbleGen, Inc., USA). This normalization method aims to make the distribution of intensities for each array in a set of arrays the same. The normalized and log-transformed intensity values were then analyzed using GeneSpring GX 7.3.1 (Agilent Technologies, USA). Fold change filters included the requirement that the genes be present in at least $150 \%$ of controls for up-regulated genes and lower than $66.67 \%$ of controls for down-regulated genes. Hierarchical clustering data were clustered into groups that behaved similarly across experiments using GeneSpring GX 7.3.1 (Agilent Technologies, USA). The clustering algorithm was Euclidean distance with average linkage.

The Database for Annotation, Visualization and Integrated Discovery (DAVID) Functional Annotation Bioinformatics Microarray Analysis (National Institute of Allergy and Infectious Diseases, $\mathrm{NIH}$, http://david.abcc.ncifcrf.gov/home.jsp) (Huang et al., 2008) was used to analyze the biological process and molecular function of the regulated genes. The lists of genes (upor down-regulated), with both the filtered fold changes and $P$ values $<0.05$, were submitted to DAVID. The functional clustering of differentially regulated genes is performed based on Gene Ontology (GO) annotation (Ashburner et al., 2000) in terms of molecular function (MF), biological process (BP) and cellular component (CC).

\section{Statistical analysis}

All statistical analyses were performed using the SAS 9.2 software package for Windows (SAS Institute, USA). The results are presented as means \pm standard error (SE). If two groups were compared, Student's $t$-test was applied. If three groups were compared, intergroup differences were analyzed by oneway analysis of variance (ANOVA) with post hoc Duncan's multiple range tests. The threshold for significance was $P<$ 0.05 .

\section{RESULTS}

Experiment 1: CGH regulates food intake and NPY mRNA levels in the hypothalamus in lean rats

In experiment 1 , normal lean SD rats were fed $\mathrm{CGH}$ as a protein source, and food intake was evaluated for 7 days. The observed changes in food intake are shown in Fig. 1A. CGH administration significantly reduced food intake compared with the intake of the control group after the second day of treatment To elucidate the changes in the expression levels of genes related to appetite in the hypothalamus, we investigated the leptin receptor (LEPR), neuropeptide-Y (NPY), and proopiomelanocortin (POMC) genes (Fig. 1B). The NPY mRNA level in the hypothalamus of the $\mathrm{CGH}$ group was significantly lower than that in the control group after 7 days of treatment. The LEPR mRNA level tended to be higher in the CGH group, and the POMC mRNA level was significantly higher in the CGH group than in the control group after 7 days of treatment.

Experiment 2: Time course of changes in lipid and glucose metabolism according to CGH administration in DIO rats

To elucidate the time course of metabolic changes, DIO rats were fed $\mathrm{CGH}$, and lipid and glucose biochemical markers were investigated at two-week intervals. After DIO induction for 13 weeks, the normal-fat diet (NF, $17 \%$ of energy) and high-fat diet (HF, $60 \%$ of energy) groups showed significant differences in body weight (Fig. 2A). The HF group showed a gradual increase in body weight during the experimental period. However, the HF-CGH group showed a time-dependent decrease in body weight, showing the same level as the NF group at 2 weeks and a significantly lower level after 5 weeks compared to the HF group. Food intake is shown in Fig. 2B. The HF group 
A

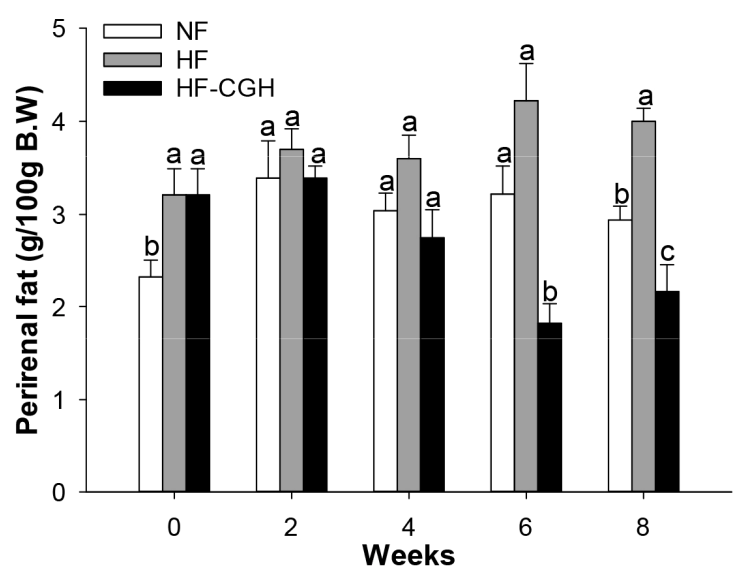

C

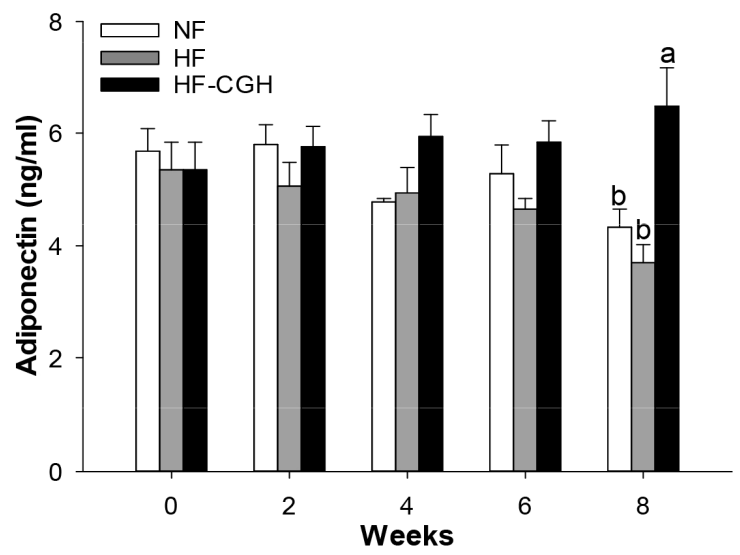

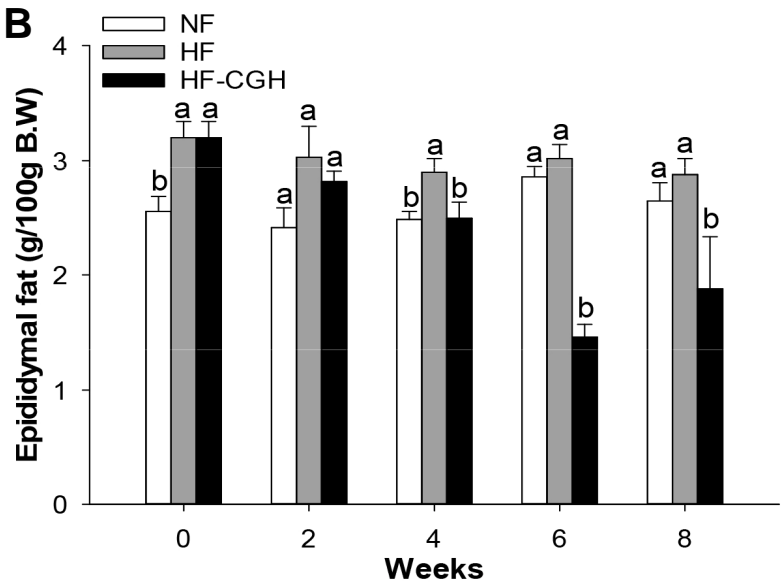

D

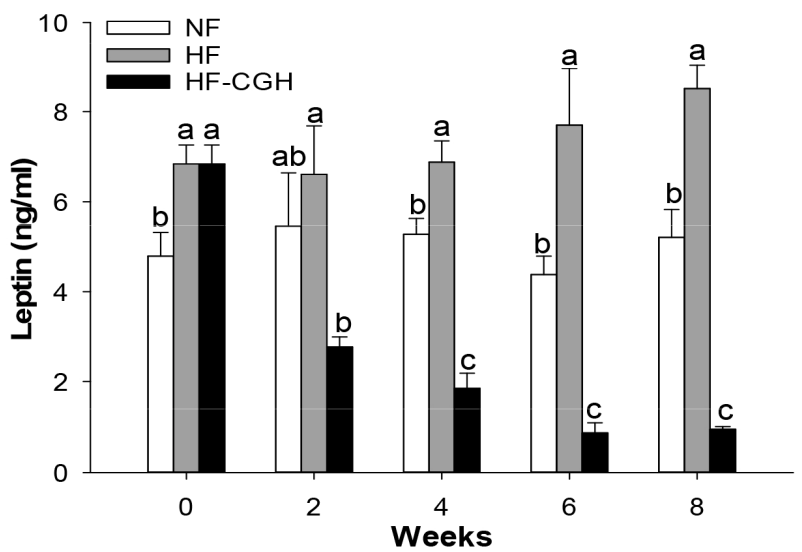

Fig. 3. Fat weights including perirenal (A) and epididymal fat (B) and adipokines including adiponectin (C) and leptin (D) profiles over 8 weeks in CGH-treated and DIO rats. Eight-week-old male SD rats were randomly divided into three groups according to body weight and fed the designated induction diet for 13 weeks; the normal-fat diet consisted of the AIN-93G diet (NF, $n=25)$, and the high-fat diet was modified from the AIN-93G diet $(H F, n=50)$. After 13 weeks, the HF group was re-randomized into 2 groups according to body weight and fed the designated experiment diet for 8 weeks: high-fat diet $(\mathrm{HF}, \mathrm{n}=25)$ or high-fat diet with $\mathrm{CGH}(\mathrm{HF}-\mathrm{CGH}, \mathrm{n}=25)$. Every 2 weeks, five rats from each group were sacrificed, and fat weight and adipokines were analyzed. Values are expressed as means \pm SE. Significant differences among the three groups were analyzed by ANOVA. Values showing a different superscript letter among the three diet groups are significantly different $(P$ $<0.05)$.

demonstrated lower food intake than the NF group, and these two groups maintained the same intake levels during the entire experiment period. However, the HF-CGH group showed significantly reduced intake levels compared to the HF group after 1 week and maintained the same level of food intake from 2 weeks until 8 weeks.

The weights of the perirenal and epididymal fat pads are shown in Figs. $3 \mathrm{~A}$ and $3 \mathrm{~B}$. The HF group showed a gradual increase in fat pad weight during the 6-week experimental period, and the NF and HF groups showed significant differences in perirenal and epididymal fat weights. However, the perirenal fat weight was significantly lower in the HF-CGH group than in the HF and NF groups at 6 weeks. Additionally, the epididymal fat weight was significantly lower in the HF-CGH group than in the HF group at 4 weeks. Plasma leptin and adiponectin levels are shown in Figs. 3C and 3D. After DIO induction for 13 weeks, leptin secreted from adipose tissue was significantly higher in the HF group than in the NF group. According to the patterns of fat mass reduction, the leptin level was also lower in the HF$\mathrm{CGH}$ group compared to the HF group after 2 weeks. In contrast, the adiponectin level was significantly increased in the HF-CGH group compared to the HF group at 8 weeks.

The HF-CGH group exhibited significantly lower plasma TG concentrations compared to the control group after 6 weeks (Fig. 4A). However, no significant differences in plasma Total-C levels were observed between the HF and HF-CGH groups (Fig. 4B). Hepatic TG, cholesterol and total lipid levels in the HF group were significantly higher than those in the NF group after inducing obesity. However, $\mathrm{CGH}$ administration lowered the hepatic TG content compared to the control group after 2 weeks (Fig. 4C) and also significantly decreased hepatic cholesterol content after 6 weeks (Fig. 4D). The HF-CGH group also showed a steady decrease in total lipids, with levels lower than those in the NF group after 4 weeks (Fig. 4E).

Increased plasma glucose levels are indicative of disturbed glucose tolerance. Fasting glucose levels were not different at 
A

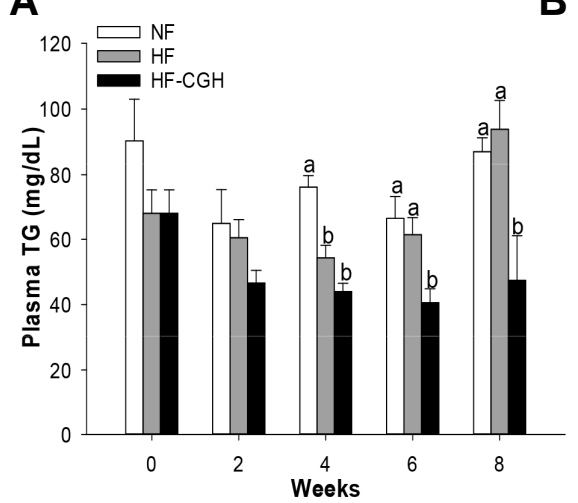

$\boldsymbol{B}$

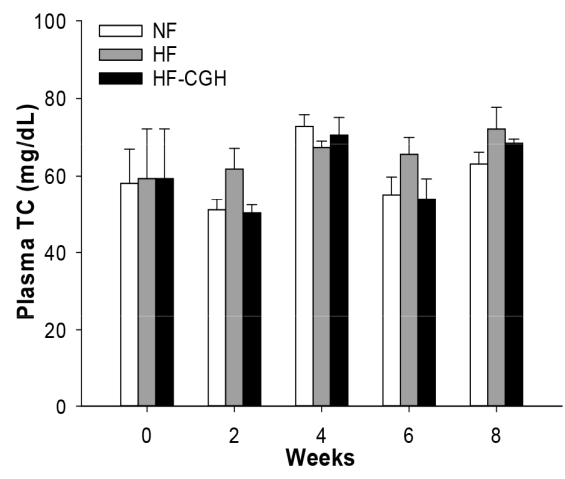

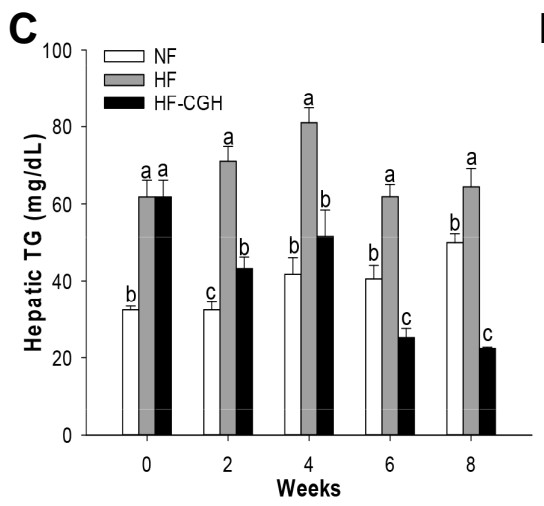

D
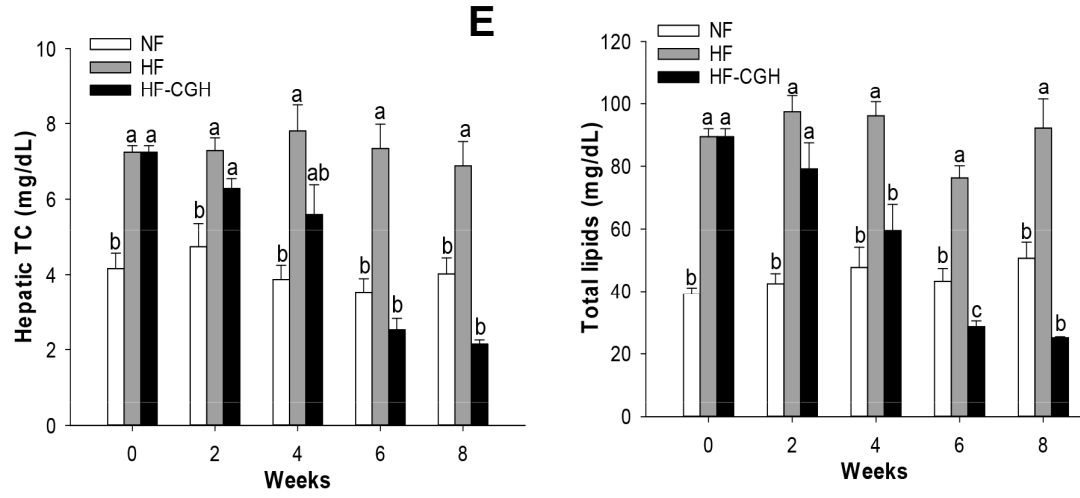

Fig. 4. Plasma $[(A)$ for plasma TG and (B) for plasma TC) and hepatic lipid ((C) for hepatic TG, (D) for hepatic TC and (E) for hepatic total lipid) profiles over 8 weeks in CGH-treated and DIO rats. Eight-week-old male SD rats were randomly divided into three groups according to body weight and fed the designated induction diet for 13 weeks; the normal-fat diet consisted of the AIN-93G diet (NF, $n=25)$, and the high-fat diet was modified from the AIN-93G diet (HF, $n=50)$. After 13 weeks, the HF group was re-randomized into 2 groups according to body weight and fed the designated experiment diet for 8 weeks: high-fat diet $(\mathrm{HF}, \mathrm{n}=25)$ or high-fat diet with CGH (HF-CGH, $n=25)$. Every 2 weeks, five rats from each group were sacrificed, and lipid profiles were analyzed. Values are expressed as means \pm SE. Significant differences among the three groups were analyzed by ANOVA. Values showing a different superscript letter among the three diet groups are significantly different $(P<0.05)$.

any time, although plasma insulin levels were significantly lower compared to the HF group after 6 weeks (Figs. 5A and 5B). Accordingly, the Homeostasis Model of Assessment - Insulin Resistance (HOMA-IR), an index of insulin resistance, was significantly improved in the HF-CGH group to the level of the normal control group at 8 weeks (Fig. 5C).

To compare the transcriptomes between the HF and HF$\mathrm{CGH}$ groups, microarray analysis was conducted using adipose tissue obtained at 8 weeks from $\mathrm{CGH}$-supplemented rats fed high-fat diets. Total RNA was obtained from the adipose tissue of rats from the HF and HF-CGH groups, and gene profiling was performed with 26,419 genes. The signal intensities of 362 genes were differently expressed between the 2 groups at levels of more than 1.5-fold or below 0.66 -fold (data not shown). The gene ontology and pathways of the differently expressed genes were analyzed using the DAVID platform to identify the functions of the differently expressed genes (DEG) in the HF and HF-CGH groups. Functional annotation clustering using DAVID revealed that the majority of genes significantly affected by $\mathrm{CGH}$ were related to the G-protein coupled receptor protein signaling pathway and sensory perception (Fig. 6). These genes included ones encoding olfactory receptors and guanine nucleotide-binding protein (Table 2). Moreover, CGH significant- ly affected the expression levels of several genes, such as low density lipoprotein receptor, coagulation factor III, and progestin and adipoQ receptor family member VIII. The genes most significantly affected were olfactory receptor 149 (Olr149) gene, with a fold value of approximately $1.70(P=0.036)$; G-protein coupled receptor 98 , with a fold value of approximately $1.67(P$ $=0.030$ ); the progestin and adipoQ receptor family member VIII, with a fold value of $0.10(P=0.002)$; and the killer cell lectin-like receptor subfamily $\mathrm{C}$, member 3 , with a fold value of $0.46(P=$ 0.034).

\section{DISCUSSION}

In the first part of this study, we investigated the effects of CGH (1.5 g/day) administration for 7 days on appetite-responsive genes in the hypothalamus of lean SD rats. We applied gavage feeding of $\mathrm{CGH}$ (1.5 g/day) to guarantee feeding consistency and avoid confounding factors associated with the poor flavor of $\mathrm{CGH}$. After 2 days of treatment, the rats' food intake was significantly reduced, and the perirenal fat pad weight had decreased significantly after 1 week (data not shown). Moreover, the major appetite-inhibiting genes POMC and LEPR were upregulated after 1 week of $\mathrm{CGH}$ administration. Neural compo- 
Metabolic Changes Affected by Corn Peptide in DIO Rats

Hyojung Lee et al.

Table 2. Fold changes of selected genes influenced by CGH in high-fat diet induced rats

\begin{tabular}{|c|c|c|}
\hline Description & $\begin{array}{l}\text { Fold } \\
\text { change }\end{array}$ & $P$-value \\
\hline \multicolumn{3}{|l|}{ Olfactory receptor activity } \\
\hline Olfactory receptor 149 & 1.70 & 0.036 \\
\hline Olfactory receptor 1250 & 1.51 & 0.002 \\
\hline Olfactory receptor 578 & 0.47 & 0.033 \\
\hline \multicolumn{3}{|l|}{ G-protein-coupled receptor protein signaling pathway } \\
\hline G-protein-coupled receptor 98 & 1.67 & 0.030 \\
\hline Olfactory receptor 1144 & 1.69 & 0.031 \\
\hline Olfactory receptor 1615 & 1.70 & 0.036 \\
\hline Olfactory receptor 602 & 0.47 & 0.033 \\
\hline \multicolumn{3}{|l|}{ Sensory perception } \\
\hline RT1, class lb, locus M5 & 0.58 & 0.014 \\
\hline Amiloride-sensitive cation channel 1 , neuronal & 1.69 & 0.031 \\
\hline \multicolumn{3}{|l|}{ Endosome membrane } \\
\hline Low-density lipoprotein receptor & 1.51 & 0.024 \\
\hline \multicolumn{3}{|l|}{ Intrinsic to membrane } \\
\hline Coagulation factor III & 1.54 & 0.002 \\
\hline cyclic nucleotide gated channel alpha 1 & 1.50 & 0.003 \\
\hline potassium large conductance calcium-activated channel, subfamily $\mathrm{M}$ beta member 3 & 1.51 & 0.038 \\
\hline ST8 alpha-N-acetyl-neuraminide alpha-2,8-sialyltransferase 4 & 1.57 & 0.011 \\
\hline solute carrier organic anion transporter family, member $1 \mathrm{a} 5$ & 1.53 & 0.023 \\
\hline progestin and adipoQ receptor family member VIII & 0.10 & 0.002 \\
\hline killer cell lectin-like receptor subfamily $\mathrm{C}$, member 3 & 0.46 & 0.034 \\
\hline
\end{tabular}

Significant differences between the HF and HF-CGH groups were evaluated by Student's $t$-test $\left({ }^{* \star} P<0.001\right)$.

nents secreted from/to the hypothalamus regulate food intake levels. One of the most important hypothalamic signal contributed to the understanding of appetite control of food is leptin. We hypothesized that $\mathrm{CGH}$ administration would decrease plasma leptin levels and hypothalamic NPY mRNA expression but increase LEPR and POMC mRNA expression. The highest levels of NPY are found in the hypothalamus although NPY is widely expressed throughout the central nervous system (CNS) (Gehlert et al., 1987). The expression and release of NPY are reduced in the fed status and increased in the fasting status, respond to changes in energy status and are reduced in feeding conditions and increased in fasting conditions (Beck et al., 1990; Kalra et al., 1991). POMC is a prohormone precursor that is cleaved into several bioactive peptides in the hypothalamus (Mercer et al., 2013). An increased level of POMC and a decreased level of NPY indicate a fasting state. According to our previous studies, rats fed $\mathrm{CGH}$ as a protein source combined with a high-fat diet demonstrated decreased obesity-related metabolic biomarkers and decreased food intake after 2 weeks of administration (Bong et al., 2010). Therefore, corn gluten hydrolysates may induce appetite control and therefore affect body weight control.

Based on these findings, in the second study, we investigated the time course of metabolic changes induced by $\mathrm{CGH}$ at intervals of 2 weeks over an 8-week period. DIO rats showed significantly higher body weight, fat mass, hepatic lipid accumulation and plasma leptin levels compared to control rats. However, after 2 weeks of $\mathrm{CGH}$ administration, body weight, food intake, hepatic TG, and plasma leptin levels were significantly decreased. Moreover, the hepatic total lipid content and epididymal fat weight were decreased after 4 weeks, and hepatic cholesterol, perirenal fat and insulin levels were significantly reduced after 6 weeks. The HOMA-IR and oral glucose tolerance test (OGTT) levels were also improved in rats fed CGH for 6 weeks, indicating an improvement in glucose tolerance. Additionally, the adiponectin levels were significantly increased after 8 weeks of treatment. The most outstanding finding of the second part of the study was the decrease in adipose tissue induced by $\mathrm{CGH}$ as early as week 2 in DIO rats, which was confirmed by a decrease in body weight, adipose tissue weight and plasma leptin. Extensive crosstalk by adipokines secreted from white adipose tissue to other organs is deeply related to multiple metabolic systems. Leptin is one of the adipokines secreted by adipose tissue, which are significantly related to the amount of body fat (Jequier, 2002). Maffei et al. (1995) clearly showed that circulating plasma leptin levels are directly proportional to body fat content in humans and animals. In this study, a significant decrease in plasma leptin levels appeared prior to 
$\boldsymbol{A}$
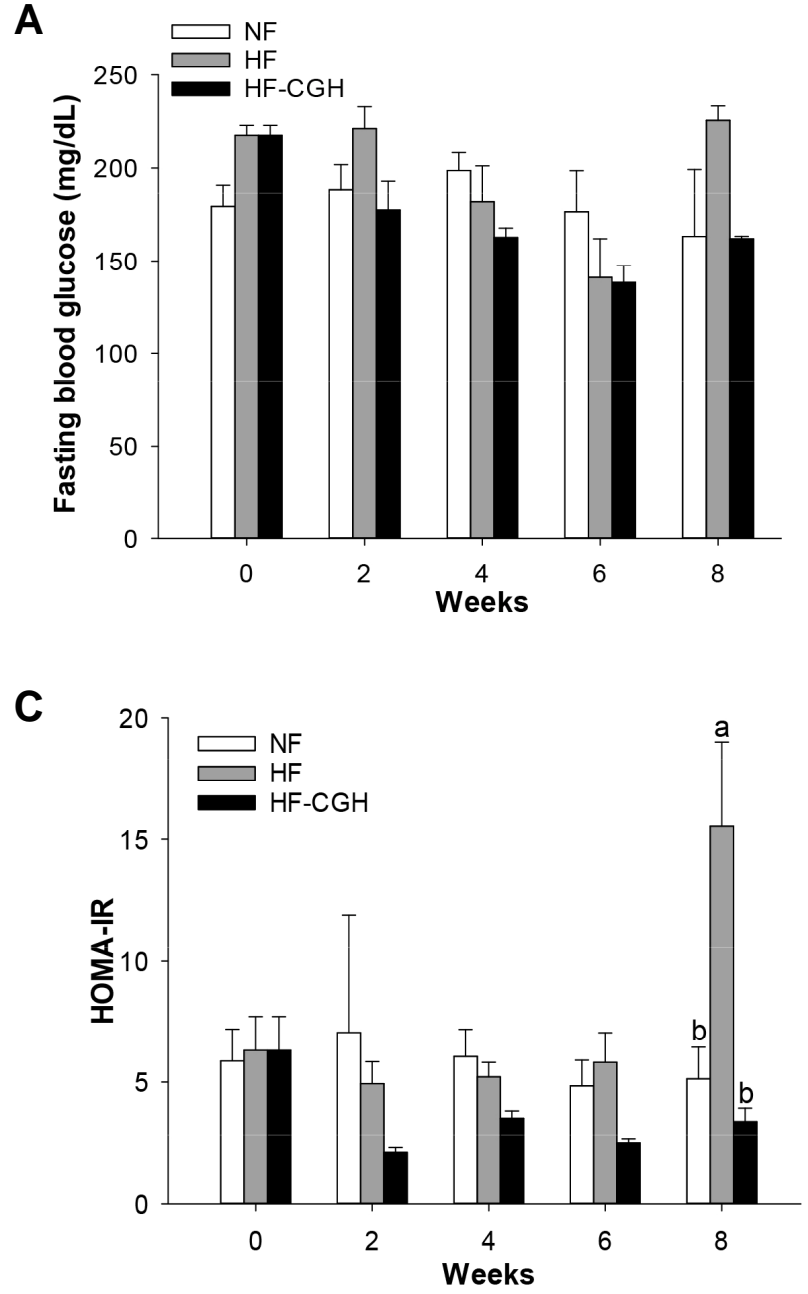

B

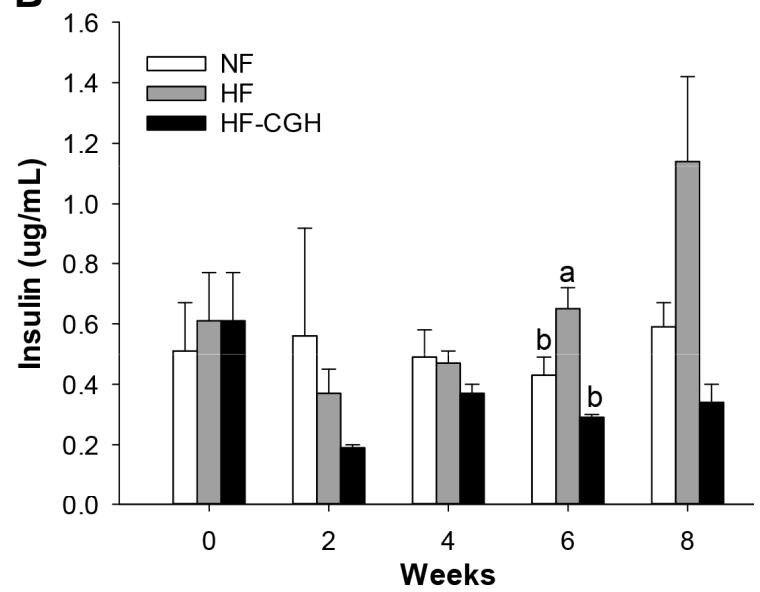

Fig. 5. Fasting glucose (A), insulin (B) and HOMA-IR (C) profiles over 8 weeks in CGH-treated and DIO rats. Eight-week-old male SD rats were randomly divided into three groups according to body weight and fed the designated induction diet for 13 weeks; the normal-fat diet consisted of the AIN-93G diet (NF, $n=25)$, and the high-fat diet was modified from the AIN-93G diet $(H F, n=50)$. After 13 weeks, the HF group was re-randomized into 2 groups according to body weight and fed the designated experiment diet for 8 weeks: high-fat diet (HF, $n=$ $25)$ or high-fat diet with CGH (HF-CGH, $\mathrm{n}=25)$. Every 2 weeks, five rats from each group were sacrificed, and fasting glucose and insulin levels were analyzed. Using glucose and insulin levels, HOMA-IR levels were calculated. Values are expressed as means \pm SE. Significant differences among the three groups were analyzed by ANOVA. Values showing a different superscript letter among the three diet groups are significantly different $(P<0.05)$.

the reduction of epididymal fat mass after 4 weeks of treatment. The plasma leptin level also corresponded to the content of hepatic TG after 2 weeks of treatment. The amelioration of lipid metabolism was preceded by improved glucose metabolism, as shown by decreased insulin and HOMA-IR levels after 6 weeks of CGH treatment. Fatty deposition in the liver were observed after only 2 weeks of HF diet compared to the normal control group. Additionally, biomarkers related to glucose metabolism including fasting glucose, impaired glucose tolerance and insulin resistance, occurred later in HF diet-fed mice (Kleemann et al., 2010; Radonjic et al., 2009).

Our findings support the conclusion that improvements in lipid metabolism may lead to the amelioration of insulin resistance as perirenal fat weights and insulin levels were significantly reduced after 6 weeks of treatment. In particular, $\mathrm{CGH}$ administration increased insulin sensitivity by lowering the plasma insulin level, which resulted in reduced leptin levels and increased adiponectin levels (Kopelman, 2000). Adiponectin affects glucose metabolism by enhancing insulin production in pancreas (Fraulob et al., 2010). In addition to its peripheral actions, adiponectin has also been known to control food intake and energy expenditure levels through its effects on the central nervous system (Kim et al., 2004). In our second experiment, the plasma adiponectin level was improved following $\mathrm{CGH}$ administration at 8 weeks of treatment.

Several microarray reports using DIO models have revealed that a high-fat diet caused a critical stimulation of inflammatory related genes and down-regulation of lipid metabolism genes (Do et al., 2011; Kim et al., 2004; Kleemann et al., 2010; Radonjic et al., 2009). In this study, we compared adipose tissue 
Metabolic Changes Affected by Corn Peptide in DIO Rats

Hyojung Lee et al.

mRNA expression between the HF and the HF-CGH groups treated for 8 weeks. According to the functional annotation
In summary, dietary CGH may help to maintain satiety under

lean conditions and reduce body fat in obese conditions. The
1. G-protein coupled receptor protein signaling pathway
2. detection of chemical stimulus involved in sensory perception of smell
3. detection of chemical stimulus involved in sensory perception
4. detection of chemical stimulus
5. sensory perception of smell
6. detection of stimulus involved in sensory perception
7. olfactory receptor activity
8. sensory perception of chemical stimulus
9. detection of stimulus
10. detection of stimulus involved in sensory perception

P-value

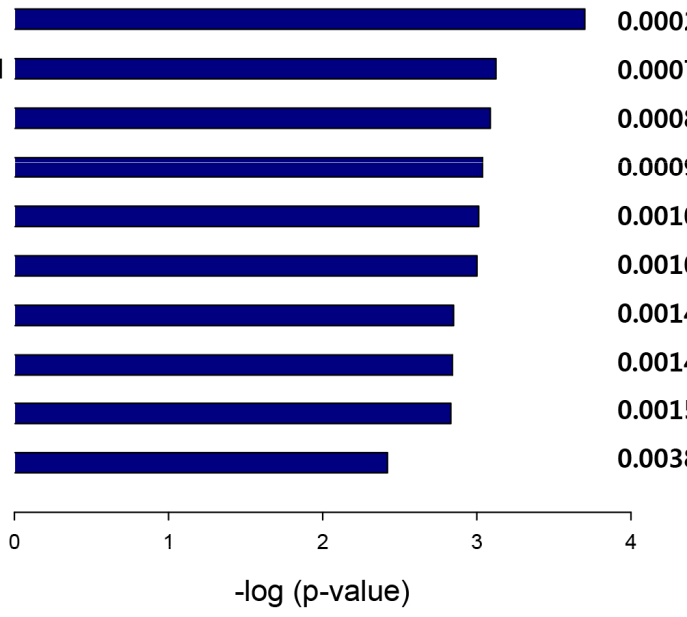

Fig. 6. Pathway analysis of white adipose tissue genes affected by $\mathrm{CGH}$ administration in high-fat diet-induced obese rats. The functional clustering of differentially regulated genes between the HF and HF-CGH groups is performed based on Gene Ontology (GO) annotation in terms of molecular function (MF), biological process (BP) and cellular component (CC). The top 10 pathways obtained are sorted based on significance. For each pathway, the $P$-value is presented.

analysis using the DAVID platform, genes associated with olfactory receptors and the G-protein coupled receptor protein as well as genes involved in lipid metabolism were significantly altered by CGH administration.

Parental high fat consumption in rats was found to downregulate olfactory genes in WAT and pancreatic islets in daughters ( $\mathrm{Ng}$ et al., 2014). In contrast, some of olfactory receptor proteins were up-regulated in WAT by high fat consumption (Joo et al., 2010). In the present study, olfactory receptors 149, $1250,90,144$ and 1615 were significantly up-regulated according to weight and fat loss; however, olfactory receptors 578 and 602 were down-regulated. Although the exact mechanisms are unclear, high fat consumption and weight loss may be related to nutrient sensing (Joo et al., 2010). In experiment 2, we revealed that rats administered $\mathrm{CGH}$ had reduced appetite, and the level of food intake was dramatically reduced at an early stage. Subsequently, lipid and glucose metabolisms were progressively ameliorated in high-fat-induced obese rats. These phenomena were in agreement with the results from experiment 1 and the transcriptome analysis. In experiment $1, \mathrm{CGH}$ administration in lean rats lead to reduced appetite and significantly reduced NPY mRNA expression after one week. In the transcriptome analysis, CGH administration in obese rats significantly affected olfactory receptor genes and the G-protein coupled receptor. NPY, which was down-regulated by $\mathrm{CGH}$ administration in experiment 1 , acts through a family of Gprotein-coupled receptors (Negroni et al., 2012). However, a limitation of the present study was that we could not validate the mRNA levels that were measured in the transcriptome analysis. Additionally, we could not determine which peptide within $\mathrm{CGH}$ controlled the appetite signal or where the signal was induced. Because $\mathrm{CGH}$ is the crude peptide mixture, we could not identify which peptide among the numerous peptide mixtures was related to appetite signaling. As a next step, the precise mechanisms and the structure of the active peptide should be elucidated. initial trigger for the reduction in obesity is likely a reduction in food intake, as CGH demonstrated a positive effect on lowering appetite levels as well as a possible anti-obesity effect during extended treatment. The relationship between leptin and appetite in the CGH group was not clearly defined in this study; however, energy-balancing hormones such as leptin and other gut hormones are clearly related to satiety. This study represents an initial step in a series of studies to reveal the effects and mechanisms of CGH on appetite control, hyperlipidemia and hyperglycemia. Further studies are required to assess the cross-talk between the hypothalamus and the gut at the molecular level in response to $\mathrm{CGH}$ administration and in clinical investigations.

\section{ACKNOWLEDGMENTS}

This study was supported by the Cooperative Research Program for Agriculture Science \& Technology Development (Project title: Research on New Functional Food Materials with Domestic Agricultural Products, Project No. PJ00845002) of Rural Development Administration, Republic of Korea.

\section{REFERENCES}

Adam, C., Williams, P., Garden, K., Thomson, L., and Ross, A. (2014). Dose-dependent effects of a soluble dietary fibre (pectin) on food intake, adiposity, gut hypertrophy and gut satiety hormone secretion in rats. PLoS One 10, e0115438-e0115438.

Aoyama, T., Fukui, K., Takamatsu, K., Hashimoto, Y., and Yamamoto, T. (2000). Soy protein isolate and its hydrolysate reduce body fat of dietary obese rats and genetically obese mice (yellow KK). Nutrition 16, 349-354.

Ashburner, M., Ball, C.A., Blake, J.A., Botstein, D., Butler, H., Cherry, J.M., Davis, A.P., Dolinski, K., Dwight, S.S., Eppig, J.T., et al. (2000). Gene ontology: tool for the unification of biology. The Gene Ontology Consortium. Nat. Genet. 25, 25-29.

Beck, B., Jhanwar-Uniyal, M., Burlet, A., Chapleur-Chateau, M., Leibowitz, S.F., and Burlet, C. (1990). Rapid and localized alterations of neuropeptide $\mathrm{Y}$ in discrete hypothalamic nuclei with feeding status. Brain Res. 528, 245-249. 
Bligh, E.G., and Dyer, W.J. (1959). A rapid method of total lipid extraction and purification. Can. J. Biochem. Physiol. 37, 911-917.

Bong, H.Y., Kim, J.Y., Jeong, H.I., Moon, M.S., Kim, J., and Kwon, O. (2010). Effects of corn gluten hydrolyzates, branched chain amino acids, and leucine on body weight reduction in obese rats induced by a high fat diet. Nutr. Res. Pract 4, 106-113.

Calder, P.C., Ahluwalia, N., Brouns, F., Buetler, T., Clement, K., Cunningham, K., Esposito, K., Jönsson, L.S., Kolb, H., Lansink, M., et al. (2011). Dietary factors and low-grade inflammation in relation to overweight and obesity. Br. J. Nutr. 106, S5-78.

Caudill, S.P., Cooper, G.R., Smith, S.J., and Myers, G.L. (1998). Assessment of current National Cholesterol Education Program guidelines for total cholesterol triglyceride, HDL-cholesterol, and LDL-cholesterol measurements. Clin Chem 44, 1650-1658.

Clark, M.J., and Slavin, J.L. (2013). The effect of fiber on satiety and food intake: a systematic review. J. Am. Coll. Nutr. 32, 200-211.

Das, U.N. (2010). Obesity: genes, brain, gut, and environment. Nutrition 26, 459-473.

Davis, J., Steinle, J., Higginbotham, D., Oitker, J., Peterson, R., and Banz, W. (2005). Soy protein influences insulin sensitivity and cardiovascular risk in male lean SHHF rats. Horm. Metab. Res. 37, 309-315

Do, G.M., Oh, H.Y., Kwon, E.Y., Cho, Y.Y., Shin, S.K., Park, H.J., Jeon, S.M., Kim, E., Hur, C.G., Park, T.S., et al. (2011). Long-term adaptation of global transcription and metabolism in the liver of high-fat diet-fed C57BL/6J mice. Mol. Nutr. Food Res. 55, S173185

Fraulob, J.C., Ogg-Diamantino, R., Fernandes-Santos, C., Aguila, M.B., and Mandarim-de-Lacerda, C.A. (2010). A mouse model of metabolic syndrome: insulin resistance, fatty liver and nonalcoholic fatty pancreas disease (NAFPD) in C57BL/6 mice fed a high fat diet. J. Clin. Biochem. Nutr. 46, 212-223.

Gehlert, D.R., Chronwall, B.M., Schafer, M.P., and O'Donohue, T.L. (1987). Localization of neuropeptide $Y$ messenger ribonucleic acid in rat and mouse brain by in situ hybridization. Synapse 1 , 25-31.

Huang, D.W., Sherman, B.T., and Lempicki, R.A. (2008). Systematic and integrative analysis of large gene lists using DAVID bioinformatics resources. Nat. Protoc. 4, 44-57.

Jequier, E. (2002). Leptin signaling, adiposity, and energy balance. Ann. NY Acad. Sci. 967, 379-388.

Joo, J.I., Kim, D.H., Choi, J.-W., and Yun, J.W. (2010). Proteomic analysis for antiobesity potential of capsaicin on white adipose tissue in rats fed with a high fat diet. J. Proteome Res. 9, 29772987.

Kalra, S.P., Dube, M.G., Sahu, A., Phelps, C.P., and Kalra, P.S (1991). Neuropeptide $Y$ secretion increases in the paraventricular nucleus in association with increased appetite for food. Proc. Natl. Acad. Sci. USA 88, 10931-10935.

Kim, S., Sohn, I., Ahn, J.I., Lee, K.H., and Lee, Y.S. (2004). Hepatic gene expression profiles in a long-term high-fat diet-induced obesity mouse model. Gene 340, 99-109.

Kim, J., Park, J., Hong, S., and Kim, M.K. (2009). Effect of corn gluten and its hydrolysate consumptions on weight reduction in rats fed a high-fat diet. Nutr. Res. Pract. 3, 200-207.

Kleemann, R., van Erk, M., Verschuren, L., van den Hoek, A.M., Koek, M., Wielinga, P.Y., Jie, A., Pellis, L., Bobeldijk-Pastorova, I., Kelder, T., et al. (2010). Time-resolved and tissue-specific systems analysis of the pathogenesis of insulin resistance. PLoS One 5, e8817.

Kopelman, P.G. (2000). Obesity as a medical problem. Nature 404, 635-643.

Layman, D.K. (2003). The role of leucine in weight loss diets and glucose homeostasis. J. Nutr. 133, 261S-267S.

Layman, D.K., and Walker, D.A. (2006). Potential importance of leucine in treatment of obesity and the metabolic syndrome. J. Nutr. 136, 319S-323S.

Maffei, M., Halaas, J., Ravussin, E., Pratley, R.E., Lee, G.H., Zhang, Y., Fei, H., Kim, S., Lallone, R., Ranganathan, S., et al. (1995). Leptin levels in human and rodent: measurement of plasma leptin and ob RNA in obese and weight-reduced subjects. Nat. Med. 1, 1155-1161.

Mercer, A.J., Hentges, S.T., Meshul, C.K., and Low, M.J. (2013) Unraveling the central proopiomelanocortin neural circuits. Front Neurosci. 7, 19

Mun, J.M., Ok, H.M., and Kwon, O. (2014). Corn gluten hydrolysate and capsaicin have complimentary actions on body weight reduction and lipid-related genes in diet-induced obese rats. Nutr. Res. 34, 458-465.

Negroni, J., Meunier, N., Monnerie, R., Salesse, R., Baly, C., Caillol, M., and Congar, P. (2012). Neuropeptide y enhances olfactory mucosa responses to odorant in hungry rats. PLoS One 7, e45266.

Ng, S.-F., Lin, R.C., Maloney, C.A., Youngson, N.A., Owens, J.A., and Morris, M.J. (2014). Paternal high-fat diet consumption induces common changes in the transcriptomes of retroperitoneal adipose and pancreatic islet tissues in female rat offspring. FASEB J. 28, 1830-1841.

Panteghini, M., Pagani, F., and Bonora, R. (1993). Clinical and analytical evaluation of a continuous enzymatic method for measuring pancreatic lipase activity. Clin. Chem. 39, 304-308

Radonjic, M., de Haan, J.R., van Erk, M.J., van Dijk, K.W., van den Berg, S.A., de Groot, P.J., Müller, M., and van Ommen, B. (2009). Genome-wide mRNA expression analysis of hepatic adaptation to high-fat diets reveals switch from an inflammatory to steatotic transcriptional program. PLoS One 4, e6646.

Schneeberger, M., Gomis, R., and Claret, M. (2014). Hypothalamic and brainstem neuronal circuits controlling homeostatic energy balance. J. Endocrinol. 220, T25-46.

Slavin, J. (2013). Fiber and prebiotics: mechanisms and health benefits. Nutrients 5, 1417-1435.

Slavin, J., and Green, H. (2007). Dietary fibre and satiety. Nutr. Bull. 32, 32-42.

Wanders, A.J., van den Borne, J.J., de Graaf, C., Hulshof, T., Jonathan, M.C., Kristensen, M., Mars, M., Schols, H.A., and Feskens, E.J. (2011). Effects of dietary fibre on subjective appetite, energy intake and body weight: a systematic review of randomized controlled trials. Obes. Rev. 12, 724-739. 